\title{
THE PYTHAGOREAN THEOREM: WHAT IS IT ABOUT?
}

\author{
ALEXANDER GIVENTAL
}

Although twenty five centuries old, the Pythagorean theorem appears vigorous and ubiquitous. A key to the distance formula in Descartes's method of coordinates, the theorem is implicitly present in all scientific models and engineering computations involving spatial relationships or trigonometry. An invisible companion to the dot-product operation, it is inherent in equations of mathematical physics and continuum mechanics, be those Laplace, NavierStokes, Maxwell or Yang-Mills. Disguised by axiomatic constructions of linear algebra and Riemannian geometry, it animates both quantum physics and Einstein's gravitation, which are otherwise so hard to reconcile. And of course, a rare day of a statistician or experimenter goes by without Euclid's nightmarefitting any three or more points into a straight line, which is accomplished by Gaussian least squares and hence is also reminiscent of Pythagoras.

Quite deservedly, therefore, the philosopher's pants are proudly displayed in middle-school textbooks to represent, perhaps, the only scientific truth circulating among the general public "with proof." The most popular one (shown in Figure 1) is very convincing indeed. Yet it pictures the whole issue as a cut-and-paste puzzle and leaves us with a feeling of disproportion: one of the most fundamental facts of nature is due to an ingenious tiling trick. The vast majority of other proofs ${ }^{1}$ are similar in nature.
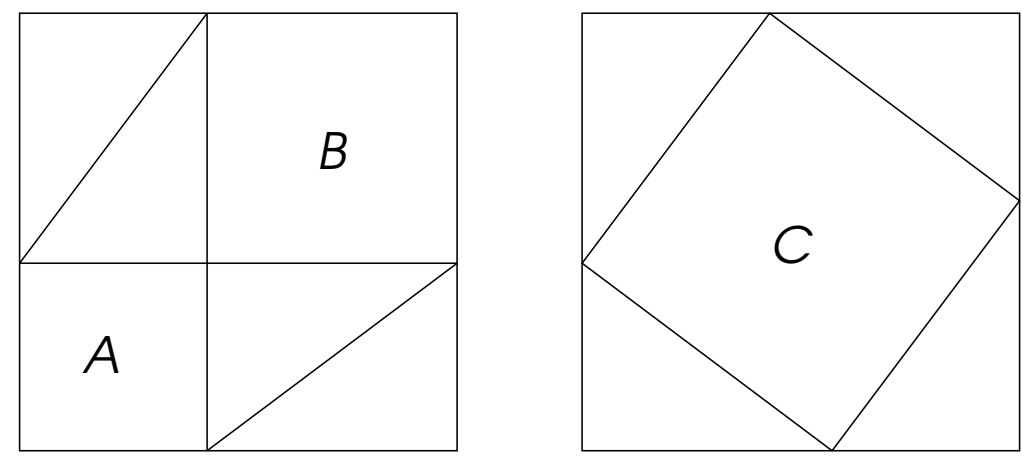

Figure $1 . A+B=C$.

\footnotetext{
${ }^{1}$ Of dozens, if not hundreds (see http://www.cut-the-knot.org/pythagoras/index.shtml and references therein).
} 
Browsing recently through Euclid's Elements [1], I found a proof of the Pythagorean theorem that some of my friends characterize as the first one they are able to comprehend. It is not only short and simple but also seems to be free of the defect just mentioned.

The Pythagorean Theorem, also known as Euclid I.47 (i.e., Proposition 47 in Book $I$ of the Elements), says that the areas of the squares built on the catheti of a right triangle add up to the area of the square built on the hypotenuse: $A+B=C$. It turns out that Book $V I$ of the Elements contains a generalization of the Pythagorean theorem that seems much less famous. Namely, Euclid VI.31 asserts (see Figure 2) that $A+B=C$ for the areas $A, B$ and $C$ of similar figures of any shape built on the sides of a right triangle. The Pythagorean theorem is clearly the special case where the shape is the square. However, the tiling argument looks hopeless when the shape is arbitrary.

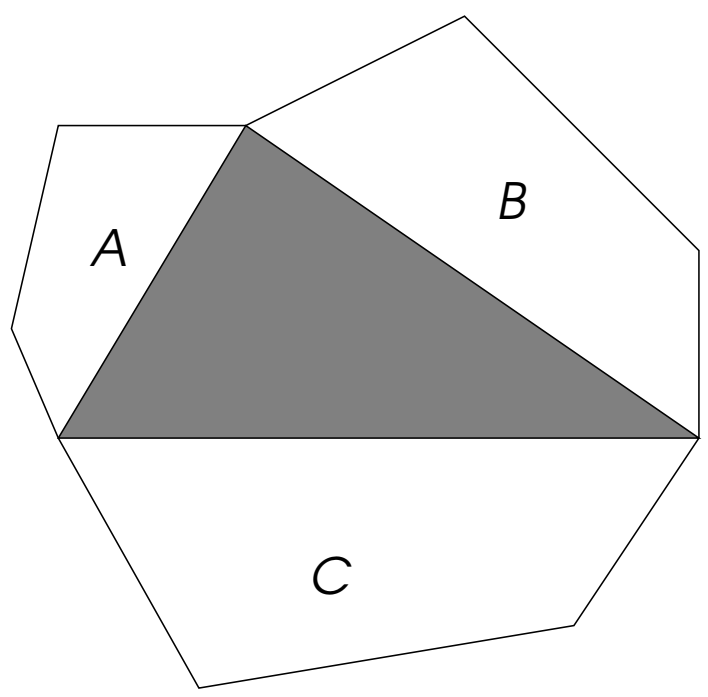

Figure 2. $A+B=C$.

On the other hand, it is not hard to figure out that the generalization, in turn, can be derived from the Pythagorean theorem since areas of similar figures scale as squares of their linear sizes. And indeed, the latter fact is contained in the Elements as Proposition VI.19 and its corollary and is quoted in Euclid's proof of the generalized Pythagorean theorem. However, Euclid uses it in order to prove the generalization in a way independent of the Pythagorean theorem; he thus reproves the latter rather than relies on it. Let us postpone the discussion of Euclid's proof and try to find one on our own.

As becomes evident from the previous remarks, it suffices to prove the generalized Pythagorean theorem for figures of one shape only. Indeed, if $a+b=c$ for areas of one shape, and areas of another shape (built on the same segments) 
are $k$ times bigger, then we have $A=k a, B=k b$ and $C=k c$, so $A+B=C$ too. We are using here the intuitively obvious fact that the ratio $k$ of the areas of two figures built on the same segment depends only on their shapes, and does not change under their simultaneous rescaling.

So, why squares? Let us instead take the shape to be the right triangle itself, of the area called $c$ (see Figure 3). The height drawn from the vertex of the right angle to the hypotenuse divides the triangle into two triangles both of which are similar to it and the areas $a$ and $b$ of which add up to $c$. That's all.
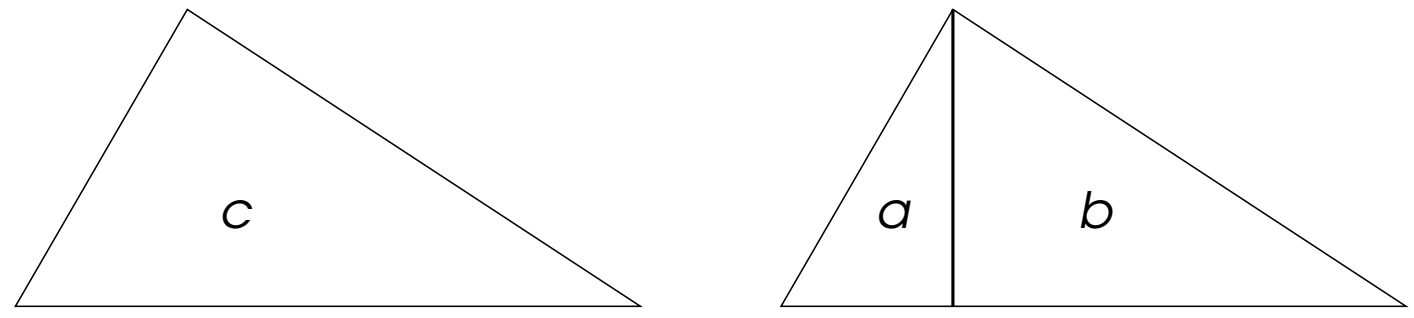

FiguRe 3. $c=a+b$.

In fact the foregoing argument is not new. Moreover, it is so close to the original proof of Proposition VI.31 that G. Polya [2] attributes it to Euclid himself. We describe Euclid's argument after the following two remarks.

First, the corresponding direct proof of the Pythagorean theorem (in its kindergarten version) now looks like this. The Three Little Pigs built three similar houses according to their sizes (Figure 4).
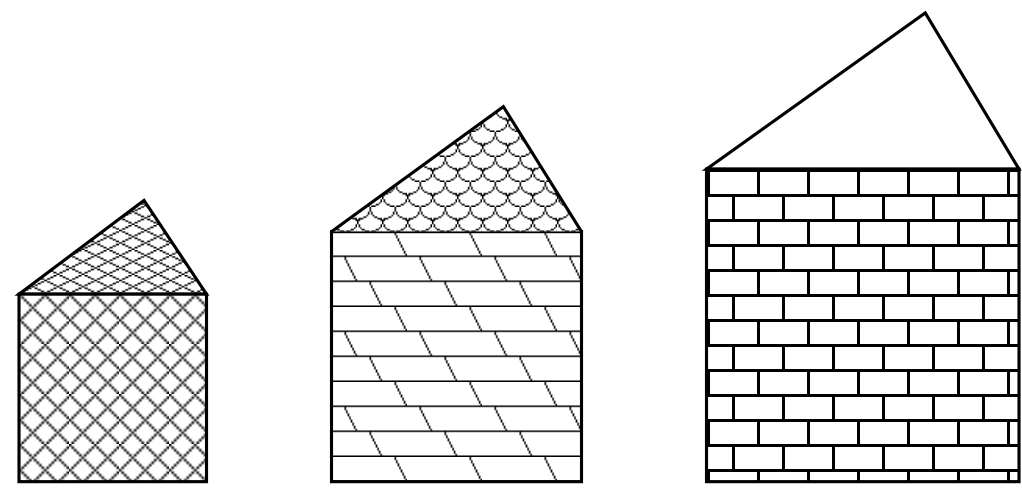

FiguRE 4. 
The two smaller attics happen to fit into the third one as in Figure 5 (in particular, this requires the attics to be right triangles).

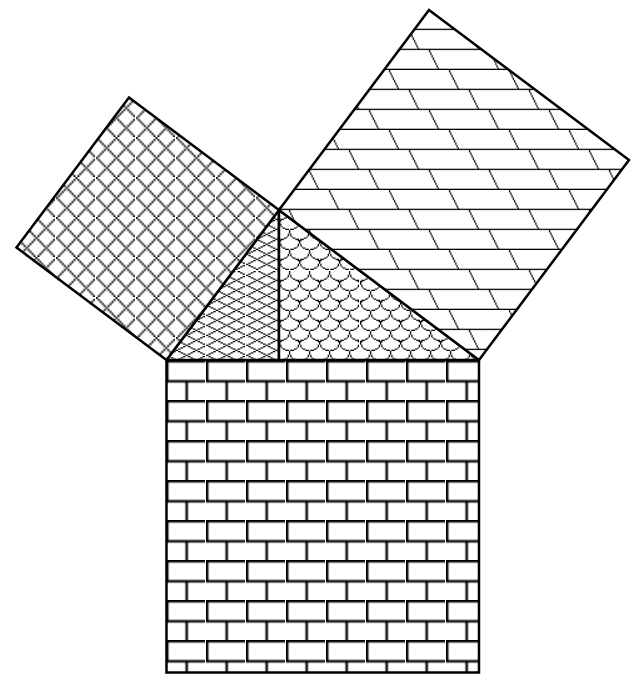

FiguRE 5 .

The three living rooms are proportionately larger than the three attics and thus the two smaller living rooms add up to the third one (Figure 6).
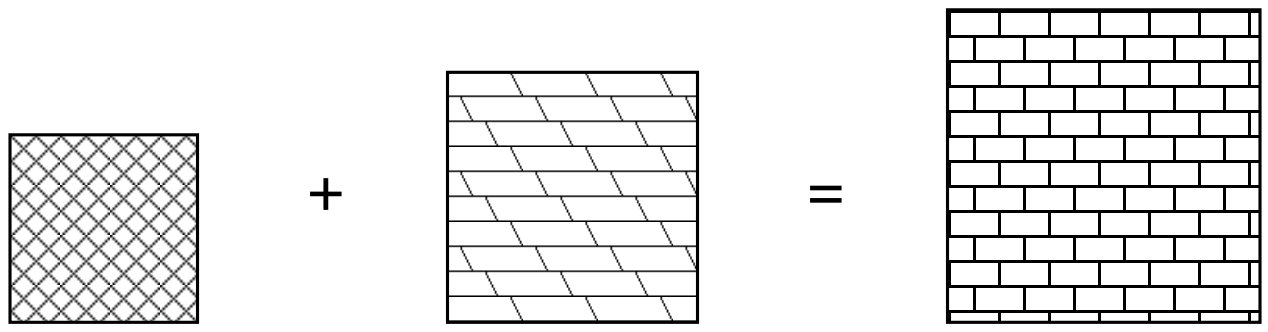

FiguRE 6.

Our second remark is somewhat more advanced. The tiling aspect in the proof of the theorem (and its generalization) is quite straightforward. As a result, the true nature of the Pythagorean theorem as a statement based on similarity is revealed. What is "similarity" after all? From the abstract point of view it is conformal isometry: an isometry of a metric space with itself equipped with a rescaled metric. This explains why the Pythagorean theorem is a genuinely Euclidean phenomenon (and not only in the historical sense of the word): among all Riemannian metrics of constant curvature only the 
Euclidean one admits nontrivial conformal isometries. Perhaps the scaling selfsimilarity property of the Euclidean geometry is that fundamental balsamic ingredient that makes the Pythagorean theorem ageless.

To appraise Euclid's original proof of the generalized Pythagorean theorem we need to realize that the power of our symbolic notation was not available to the ancient Greek mathematicians. In particular, numbers in the Elements are understood as ratios of geometrical magnitudes. The precise meaning is established by Definition V.5 (see [1, p. 114]):

Magnitudes are said to be in the same ratio, the first to the second and the third to the fourth, when, if any equimultiples whatever be taken of the first and the third, and any equimultiples whatever of the second and the fourth, the former equimultiples alike exceed, are alike equal to, or alike fall short of, the latter equimultiples respectively taken in corresponding order.

Translating this to modern terminology, two real numbers $\alpha$ and $\beta$ are called equal when, if any whole numbers $n$ and $m$ whatever be taken, $n \alpha$ and $n \beta$ both exceed, are both equal to, or both fall short of $m$. This definition was revived in the nineteenth century by Dedekind [3] who proposed to regard a real number $\alpha$ as a cut: a partition of all fractions $m / n$ according to the property of exceeding, being equal, or falling short of $\alpha$, and respectively to call two real numbers equal when the corresponding partitions are "alike" (i.e., when they coincide).

Furthermore, in order to express the square $\alpha^{2}$ of a number, the concept of the duplicate ratio is introduced ([1, Definition V.9, p. 114]):

When three magnitudes are proportional [that is the first is to the second what the second is to the third-A.G.], the first is said to have to the third the duplicate ratio of that which it has to the second.

For instance, the scaling law for areas of similar triangles is formulated in Proposition VI.19 this way: similar triangles are to one another in the duplicate ratio of the corresponding sides. The corollary ("porism") to this proposition extends this property to arbitrary figures. This reduces the generalized Pythagorean theorem to the identity $\alpha^{2}+\beta^{2}=1$, where $\alpha$ and $\beta$ are ratios of the catheti of a right triangle to its hypotenuse.

Euclid's proof of the theorem is shown in Figure 7 and is also based on the property of the familiar height to divide the right triangle into two triangles similar to it. 
Here it is: the hypotenuse is composed of two segments that have to it the duplicate ratios of those that the corresponding catheti have to it.

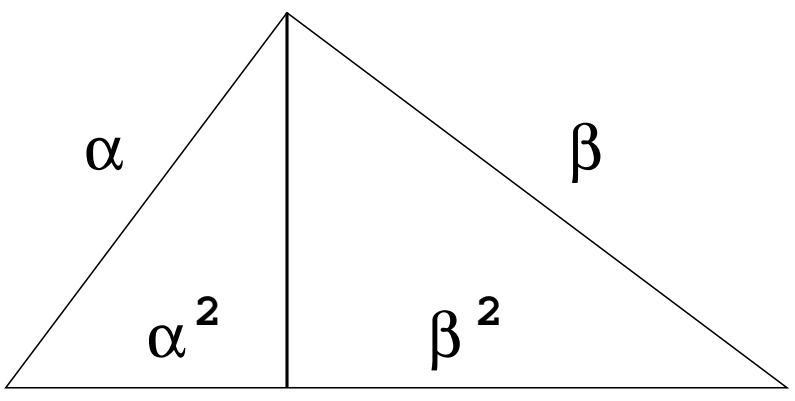

1

FiguRE 7. $\alpha^{2}+\beta^{2}=1$.

The similarity property is employed here in precisely the same way as in Figure 3, and the tiling portion of the former argument is replaced here with its one-dimensional counterpart. Apparently, the ancient Greek mathematicians understood the essense of the subject more clearly than we tend to think they did.

\section{REFERENCES}

[1] Euclid, The Thirteen Books of the Elements (trans. with introduction and commentary by T. L. Heath), 2nd ed., vol. 1 (Books I-II), vol. 2 (Books III-IX), vol. 3 (Books X-XIII), Dover, New York, 1956.

[2] G. Polya, Mathematics and Plausible Reasoning, vol. 1 (reprint of the 1954 original) Princeton University Press, Princeton, 1990.

[3] R. Dedekind, Essays on the Theory of Numbers, I:Continuity and Irrational Numbers, II:The Nature and Meaning of Numbers (trans. W. W. Beman) Dover, New York, 1963.

Department of Mathematics, University of California Berkeley, Berkeley CA 94720

givental@math.berkeley.edu 